\title{
Blood pressure variability and its implications in people of African descent
}

\author{
Ifeoma I Ulasi, Benedict C Anisiuba and Chinwuba K Ijoma \\ Hypertension Research (2011) 34, 1076-1077; doi:10.1038/hr.2011.138; published online 18 August 2011
}

$\mathrm{T}$ Techniques that assess systolic and diastolic blood pressure (BP) values in ambulant subjects have revealed that BP fluctuates spontaneously throughout the day. ${ }^{1}$ Blood pressure variability has pathophysiological implications. The values may vary by more than $50 \mathrm{~mm} \mathrm{Hg}$ in a day. These fluctuations are due to pressor and depressor episodes, and exhibit diurnal variation with high daytime and low nighttime values.

Elevated BP is the most common and modifiable risk factor for cardiovascular disease. $^{2}$ Clinicians must recognize that measured BP is an intrinsically variable hemodynamic phenomenon. Measured BP remains variable even after correcting for faulty equipment or poor technique. ${ }^{3}$

Recent studies have revealed distinct pathophysiological patterns of BP variability and reactivity that are clinically significant. These patterns are related to the modulation of sympathoadrenal output, to vascular reactivity and to behavioral aspects. ${ }^{4}$ Sympathoadrenal output is modified by a variety of environmental stressors, baroreceptor reactivity and hypothalamic factors. These modifiers are affected by age, hypertension, genetic factors, angiotensin II levels and disease state. For example, normotensive individuals with a family history of hypertension have decreased baroreceptor activity, which contributes to BP variability. The study by Schutte et al. ${ }^{5}$ did not indicate any family history of hypertension in their subjects.

Dr II Ulasi and CK Ijoma are at the Renal Unit, Department of Medicine, College of Medicine, University of Nigeria Teaching Hospital, Ituku-Ozalla, Enugu 400001, Nigeria and BC Anisiuba is at the Cardiology Unit, Department of Medicine, College of Medicine, University of Nigeria Teaching Hospital, Ituku-Ozalla, Enugu, Nigeria.

E-mail: ifeomaulasi@yahoo.co.uk
They studied BP variability in hypertensive and normotensive Caucasians and people of African descent in South Africa. The prevalence of hypertension is higher in people of African descent than in Caucasians. In addition, normotensive people of African descent are more likely to have a family history of hypertension compared with Caucasians. Therefore, we hypothesized that this pattern of family history contributes to a higher incidence of BP variability in subjects of African descent.

Vascular reactivity is another pathophysiological mechanism that contributes to BP variability. Aging, hypertension, hypercholesterolemia and insulin resistance leads to increased vasoconstriction when associated with mental stress and dynamic exercise and subsequently promotes an increased BP response.

Behavioral aspects such as anger, hostility, poor coping abilities in stressful situations, poor social support and racial differences can increase BP reactivity. Blood pressure reactivity to mental stress is greater in people of African descent than in Caucasians. Blood pressure also tends to increase in individuals who move from rural to urban or semiurban settings. Apparent racial differences in $\mathrm{BP}$ reactivity to environmental stress have been detected by many studies, ${ }^{6,7}$ including that of Schutte et al., ${ }^{5}$ and these differences may be explained by different genetic traits. However, negative environmental factors such as diet, weight gain, physical inactivity, high levels of anger and hostility, reduced social support and an imbalance between environmental stress and an individual's coping abilities are also significant contributing factors. ${ }^{4}$ A study by Li et al. ${ }^{8}$ has shown that African Americans have greater BP variability compared with European Americans.
However, Li et al. ${ }^{8}$ have demonstrated that in a comprehensive model, which included body mass index, waist circumference and the father's educational level, the ethnic differences in systolic BP variability are no longer significant.

The mechanisms involved in BP variability have not been clearly elucidated. However, several factors such as humoral and neural factors (central or reflex) and behavioral mechanisms have been implicated. ${ }^{9}$ Various humoral factors participate in the mechanisms of BP variability; these include nitric oxide, angiotensin and insulin. Studies have revealed a relationship between $\mathrm{BP}$ variability and resting sympathetic activity, ${ }^{10}$ which progressively increases from a normotensive to a hypertensive state. An enhanced cardiovascular drive may also be implicated in the increased BP variability noted in essential hypertension.

Behavioral factors are known to increase or decrease BP values and variability. Although dynamic physical exercises and emotional stress increase BP fluctuations, sleep and digestion decrease them. The balance between positive and negative behavioral factors is the mechanism underlying white coat hypertension. Blood pressure fluctuations are also caused by changes in respiration and baroreflex mechanisms that are mediated by an autonomic drive.

Blood pressure variability is usually assessed by calculating the s.d. of 24 -h systolic, diastolic and mean arterial pressure using intra-arterial BP monitoring in ambulant subjects. The $24-\mathrm{h}$ s.d. is estimated from beat-to-beat tracing, which is usually captured from the automatic BP monitoring system. Beat-to-beat finger BP values can be used to estimate 24-h BP variability with minimal error, especially if diastolic and mean arterial pressures are used. 
In addition to 24 -h variability, BP variability within 1 day, which is termed as diurnal variation, is evaluated. The sensitivity of the detection methods allows the capture of momentary fluctuations. Interseasonal BP fluctuations have been demonstrated by the PAMELA study, ${ }^{11}$ which shows that BP values are usually lower during the summer months and higher during the winter months.

When using out-of-office BP monitoring, different patterns of BP variability are discernible, such as circadian rhythms, early morning BP surges, white coat hypertension and masked hypertension. The diurnal variation of BP, which decreases during the night and increases during the day, is called the circadian rhythm. ${ }^{12}$ Abnormal circadian rhythms include non-dippers, ${ }^{13}$ extreme dippers with or without early morning surges and risers.

Studies have documented the inadequacy of isolated $\mathrm{BP} /$ clinical measurements to realistically depict BP changes during normal daily life. Many previous studies have documented the relevance of BP variability and its relationship to target organ damage in hypertension. Parati et al. ${ }^{14}$ have shown that target organ damage resulting from hypertension is more closely related to 24-h ambulatory systolic or diastolic BP compared with clinical measurements using a sphygmomanometer. The 24-h mean BP value and the 24-h BP variability correlates with organ damage. This finding has been confirmed for target organ damage such as vascular hypertrophy, left ventricular hypertrophy, systolic and diastolic dysfunction, cerebral infarction, retinopathy and renal damage. Therefore, the assessment of BP variability is an important research tool for exploring the pathophysiology of hypertension in clinical settings and for evaluating the effects of drug therapy.

The predictive value of BP variability has been explored by various studies. Frattola et al. ${ }^{15}$ conducted a study with a cohort of 73 patients with hypertension who were followed up after 7 years. Frattola et al. ${ }^{15}$ noted that patients who had higher BP variability with similar 24-h mean BP values had more end-organ damage.

The correlation of BP variability with target organ damage underscores the implication of the findings of Schutte et al., ${ }^{5}$ which identify BP variability as a predictive factor of left ventricular hypertrophy in normotensive subjects of African descent. Hypertension is more common in people of African descent than in Caucasians. In addition, hypertension is more frequently associated with complications. In most settings, hypertensive patients of African descent present late and are diagnosed late. Therefore, treatment for hypertension is delayed. Developing countries have fewer resources for the management of patients. Therefore, the identification of early markers of subclinical organ damage has significant prognostic implications.

The clinical pattern of BP variability should be considered when assessing cardiovascular risk. The relationship between BP variability and cardiovascular risk presents a paradox. White coat hypertension is associated with less risk compared with true hypertension. Increased BP reactivity occurs in the setting of high cardiovascular risk (such as hypercholesterolemia, dysglycemia or hypertension). Early morning BP surges and masked hypertension are associated with high cardiovascular risk. On the basis of these findings, BP variability and the clinical pattern of BP variability should be identified to determine the cardiovascular risk.

$\mathrm{BP}$ variability also has implications in the diagnosis of hypertension, in the assessment of response to antihypertensive medications and in research that mainly uses office BP because of the low cost and ease of the procedure. Elevated BP during an office visit may be a chance variation and does not necessarily indicate hypertension or poor response to treatment. Wide BP variations in the elderly will necessitate a larger sample size in any research involving the elderly population.

Using the findings of Shutte et al., ${ }^{5}$ we are interested in the implications of the association between systolic BP variability and target organ damage in normotensive patients. In addition, these implications may alter the current definition of BP. We suggest that family histories of hypertension and behavioral, environmental or other unidentified factors may contribute to BP variability.

This report is a stimulus for further research, especially in people of African descent, to unravel the implications of the association between systolic BP variability and target organ damage in normotensive subjects.

\section{CONFLICT OF INTEREST}

The authors declare no conflict of interest.

1 Mancia G, Ferrari A, Gregorini L, Parati G, Pomidossi G, Bertinieri G, Grassi G, di Rienzo M, Pedotti A, Zanchetti A. Blood pressure and heart rate variabilities in normotensive and hypertensive human beings. Circ Res 1983; 53: 96-104.

2 Peter M Rothwell, Sally C Howard, Eamon Dolan, Eoin O'Brien, Joanna E Dobson, Bjorn Dahlöf, Peter S Sever, Neil R Poulter. Prognostic significance of visitto-visit variability, maximum systolic blood pressure, and episodic hypertension. Lancet 2010; 375: 895-905.

3 Musini VM, Wright JM. Factors affecting blood pressure variability: lessons learned from two systematic reviews of randomized controlled trials. PLOS ONE 2009; 4: e5673.

4 Izzo Jr JL. Blood pressure variability and reactivity. In: Hypertension Primer, 4th ed. Lippincot Williams and Wilkins: Philadelphia, 2008

5 Schutte AE, Schutte R, Huisman HW, van Rooyen JM, Fourie CMT, Malan NT, Malan L. Blood pressure variability is significantly associated with ECG left ventricular mass in normotensive Africans: The SABPA Study. Hypertens Res 2011; 34: 1127-1134.

6 Lepore SJ, Revenson TA, Weinberger SL, Weston P, Frisina PG, Robertson $\mathrm{R}$, Portillo MM, Jones $\mathrm{H}$, Cross W. Effects of social stressors on cardiovascular reactivity in black and white women. Ann Behav Med 2006; 31: 120-127.

7 Paul J Mills PJ, Berry CC, Dimsdale JE. Race affects the decline in blood pressure with hospitalization. $\mathrm{Am} \mathrm{J}$ Hypertens 1997; 10: 1091-1096.

8 Li Z, Snieder H, Su S, Harshfield GA, Treiber FA, Wang X. A longitudinal study of blood pressure variability in African-American and European American youth. J Hypertens 2010; 28: 715-722.

9 Mancia G, Grassi G. Mechanisms and clinical implications of blood pressure variability. J Cardiovasc Pharmacol 2000; 35(7 Suppl 4): S15-S19.

10 Mancia G. Bjorn Folkow Award Lecture. The sympathetic nervous system in hypertension. J Hypertens 1997; 15: 1553-1565.

11 Sega R, Cesana G, Bombelli M, Grassi G, Stella ML, Zanchetti A, Mancia G. Seasonal variations in home and ambulatory blood pressure in the PAMELA population. Pressione Arteriose Monitorate E Loro Associazioni. J Hypertens. 1998; 16: 1585-1592.

12 Pickering TG. 2006)\#\#Ambulatory blood pressure monitoring. N Engl J Med 2006; 354: 2368-2374.

13 Hoshide Y, Kario K, Schwartz JE, Hoshide S, Pickering TG, Shimada K. Incomplete benefit of antihypertensive therapy on stroke reduction in older hypertensives with abnormal nocturnal blood pressure dipping (extremedippers and reverse-dippers). Am J Hypertens 2002; 15: 844-850.

14 Parati G, Pomidossi G, Albini F, Malaspina D, Mancia G. Relationship of 24-h blood pressure mean and variability to severity of target organ damage in hypertension. J Hypertens 1987; 5: 93-98.

15 Frattola A, Parati G, Cuspidi C, Albini F, Mancia G. Prognostic value of $24-\mathrm{h}$ blood pressure variability. J Hypertens 1993; 11: 1133-1137. 